\title{
Disability assessment using Google Maps
}

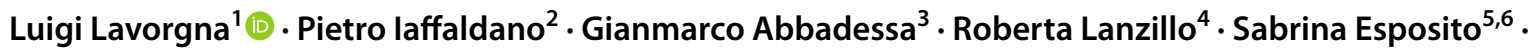 \\ Domenico Ippolito ${ }^{7}$. Maddalena Sparaco ${ }^{8}$. Simone Cepparulo ${ }^{8}$. Giacomo Lus $^{3} \cdot$ Rosa Viterbo $^{2} \cdot$ Marinella Clerico $^{9}$. \\ Francesca Trojsi ${ }^{10}$. Paolo Ragonese ${ }^{11}$. Giovanna Borriello ${ }^{12}$. Elisabetta Signoriello ${ }^{3}$. Raffaele Palladino ${ }^{13}$. \\ Marcello Moccia ${ }^{4} \cdot$ Francesco Brigo $^{14} \cdot$ Maria Troiano $^{2} \cdot$ Gioacchino Tedeschi $^{15} \cdot$ Simona Bonavita $^{8}$
}

Received: 25 January 2021 / Accepted: 7 June 2021 / Published online: 17 June 2021

(c) Fondazione Società Italiana di Neurologia 2021, corrected publication 2021

\begin{abstract}
Objectives To evaluate the concordance between Google Maps ${ }^{\circledR}$ application (GM®) and clinical practice measurements of ambulatory function (e.g., Ambulation Score (AS) and respective Expanded Disability Status Scale (EDSS)) in people with multiple sclerosis (pwMS).

Materials and methods This is a cross-sectional multicenter study. AS and EDSS were calculated using GM® and routine clinical methods; the correspondence between the two methods was assessed. A multinomial logistic model is investigated which demographic (age, sex) and clinical features (e.g., disease subtype, fatigue, depression) might have influenced discrepancies between the two methods.

Results Two hundred forty-three pwMS were included; discrepancies in AS and in EDDS assessments between GM® and routine clinical methods were found in 81/243 (33.3\%) and 74/243 (30.4\%) pwMS, respectively. Progressive phenotype (odds ratio $[\mathrm{OR}]=2.8 ; 95 \%$ confidence interval $[\mathrm{CI}] 1.1-7.11, p=0.03)$, worse fatigue $(\mathrm{OR}=1.03 ; 95 \% \mathrm{CI} 1.01-1.06, p=0.01)$, and more severe depression $(\mathrm{OR}=1.1 ; 95 \% \mathrm{CI} 1.04-1.17, p=0.002)$ were associated with discrepancies between $\mathrm{GM} \otimes$ and routine clinical scoring.

Conclusion $\mathrm{GM} ®$ could easily be used in a real-life clinical setting to calculate the AS and the related EDSS scores. GM® should be considered for validation in further clinical studies.
\end{abstract}

Keywords Digital health $\cdot$ e-Health $\cdot$ Google Maps $\cdot$ Ambulatory disorders

\section{Introduction}

In response to the coronavirus disease 2019 pandemic, many hospitals have implemented restriction policies to hospitals access [1]. These policies, as well as, social distancing, limitations for caregivers' access to outpatient clinic, mobility restrictions measures, fear of contagion, and quarantining of health-care providers, have drastically reduced physician in-person visits during the pandemic [1]

This unprecedented situation has raised the need to establish an efficient service of telemedicine to replace

Luigi Lavorgna and Pietro Iaffaldano contributed equally to this work.

Luigi Lavorgna

luigi.lavorgna@policliniconapoli.it

Extended author information available on the last page of the article face-to-face visits, monitor disease progression, and manage complications as soon as possible $[2,3]$

Recently, the reliability of telemedicine tools as alternative to EDSS quantified through clinical in-person visits has been explored in several studies [2, 4, 5]. Bove and colleagues generated a telemedicine-based MS disability examination using a digital tool and a basic neuro kit and tested it against an EDSS assessment performed by a neurologist [4]. The authors showed that disability evaluation in MS is feasible using telemedicine without an aid at the patient's location [4]. Further, a pilot study evaluated the reliability of a wearable biosensor to assess pwMS walking ability remotely, revealing that motor parameters derived from the accelerometer could be a reliable measure of motor disability in pwMS, suggesting it could be a useful tool to monitor MS patients' endurance remotely [5].

Evaluation of patient's walking ability is an important part of neurological examination [6], i.e. the maximum 
distance patients can walk without rest or the distance they can walk until symptoms occur (maximum walking distance, MWD) [7]. In clinical practice, due to time and space constraints, physicians frequently rely on patients' responses on the distance covered. However, several studies have shown the inaccuracy of distance estimates and therefore the unreliability of disability assessment in clinical routine [8].

About $40-75 \%$ of people with MS (pwMS) have impairment of the walking ability [9]. Although all deficits contribute to overall disability, gait impairment weighs more than other functional systems (FS) in disability assessment [6] The Ambulation Score (AS) of the Expanded Disability Status Scale (EDSS) [6] is the most widely used method to assess gait impairment in pwMS; in clinical practice, the MWD to rate AS is assessed by asking pwMS how long they could walk without rest, and consequently, the AS and therefore the EDSS rating may be affected by subjective, hence possibly inaccurate, MWD estimate and reporting [10].

In the last 10 years, digital technology has facilitated exact distance measurement [11]. Google Maps ${ }^{\circledR}(\mathrm{GM} \circledast)$ has revolutionized distance calculation by simply using a digital device (e.g., a smartphone) and an internet connection. We used the GM® application to evaluate the MWD in pwMS and verified its correspondence with the estimates reported by these patients and the objective measures assessed by neurologists, in order to determine whether $\mathrm{GM} ®$ provides an accurate and accessible method to evaluate walking distances in pwMS. The aim of the study was to investigate whether GM® might be used as an objective tool to evaluate the AS in pwMS and its potential implications on the accuracy of EDSS scoring.

\section{Methods}

\section{Study population}

This cross-sectional multicenter study was conducted in five MS clinical centers in Italy (two centers in Naples, one in Rome, Bari, and Palermo). PwMS were consecutively enrolled from February to May 2019. The inclusion criteria were diagnosis of clinically isolated syndrome or confirmed MS according to McDonald criteria 2010; EDSS score $\leq 6$; mental functional system $\leq 1$; disease duration (DD) $\geq 1$ year; and agreement to display a route on GM® . Exclusion criteria were ongoing relapse or steroid treatment during the 6 months preceding enrollment; any other diseases or conditions which might influence ambulation (e.g., fractures, recent surgery, etc.); and patients who ask to display a route on $\mathrm{GM}{ }^{\circledR}$ which was either uphill or downhill.

We did not include a control group as the MS-specific metrics do not apply to putative healthy controls. Indeed, while the MWD could be assessed in healthy subjects, we evaluated the correlation between the measures relying on AS and EDSS scores that do not apply to putative healthy controls.

\section{Study variables}

Demographic (age, sex, education) and clinical (DD, disease phenotype, ongoing disease-modifying therapies, DMTs) data were collected. Fatigue Severity Scale (FSS) [12] and Patient Health Questionnaire-9 (PHQ-9) [13] were used to evaluate fatigue and depression, respectively. For EDSS evaluation, pwMS were asked to estimate their MWD according to routine practice as reported above [1]. Patients' perceived AS (pAS) and, consequently, the perceived EDSS score (pEDSS) were calculated based on the MWD reported by the patients. During the enrollment visit, a neurologist blinded to the previously collected data illustrated to the patients how to use GM® on a smartphone or a hospital computer. Using GM® satellite imagery (with the street maps and $360^{\circ}$ panoramic Street View) and fixing their home address as starting point, all the recruited patients were asked to indicate a route usually followed from their home and the distance they could walk (adopting a moderate and regular walking pace) without stopping or needing any help. This was done to identify the arrival point on GM ${ }^{\circledR}$ and then measure the start-arrival distance. Based on the MWD evaluated by GM®, the GM®AS (gmAS) and the GM®EDSS (gmEDSS) were calculated.

In 75 pwMS, a blinded neurologist measured the MWD by walking the $20 \mathrm{~m}$. The MWD objectively measured was used to calculate the objectiveAS (obAS) and the objective EDSS (obEDSS) to test the consistency of the pAS and with the pEDSS, respectively. Since EDSS is heavily influenced by walking ability particularly in the mid-range scores (4.0-6.0) [14-16], the overall study population was stratified into two subgroups according to mild (pEDSS $\leq 3.5$ ) or moderate $(4 \leq$ pEDSS $\leq 6)$ disability.

\section{Standard protocol approvals, registrations, and patient consent}

According to the good clinical practice and the Declaration of Helsinki, all pwMS gave their written consent to participate in the study, which was approved by the Ethical Committee of each MS center involved.

\section{Statistical analysis}

Given the exploratory nature of this study, no sample size calculation was performed [17, 18]. Normal distribution of variables of interest was checked with graphical and statistical approaches. Continuous variables were described both as mean (M) and standard deviation (SD), median, 
and range interquartile (Q1-Q3); categorical variables were described as frequency and percentage. Descriptive statistics were performed where appropriate. Specifically, two-group comparisons were performed using the Student's $t$ test, the Mann-Whitney U test, and the Chi-square or Fisher's exact test. Comparisons between multiple groups were performed employing the analysis of variance (ANOVA) or nonparametric Kruskal-Wallis test. Correlation analyses were performed using the Spearman correlation test [19]. To calculate agreement in measures estimated by the neurologist, pwMS, or GM® service, the intraclass correlation coefficient (ICC) [20] was employed using a two-way mixed effect model. ICC estimates and their $95 \%$ confidence intervals were calculated. The two-way mixed effect model was chosen as the neurologists who were blinded to the pAS and pEDSS scores identified to acquire the objective MWD were chosen before commencing the study [21].

The following two new subgroups were identified:

1. pwMS with pEDSS correspondent to gmEDSS

2. pwMS with pEDSS different (higher/lower) from gmEDSS

Univariate and multivariate logistic regression models were applied to evaluate whether age, level of education, DD, clinical phenotype, FSS score, and PHQ-9 score might have influenced the discrepancy between the pEDSS and the gmEDSS. Results were presented as weighted Kappa, odds ratio (OR), and corresponding $95 \%$ confidence interval (CI), as appropriate. A $p$ value of $<0.05$ was considered statistically significant. Model goodness of fit was assessed using the Akaike information criterion (AIC) and the Bayesian information criterion (BIC).

Statistical analysis was performed by using SPSS software (SPSS, version 22.0; SPSS Inc., Chicago, IL). The only missing data were about the educational level for 13 patients. According to a previous study, outcomes with $<20 \%$ missing data were not excluded from the final analysis [22].

\section{Results}

\section{Sample description}

Two hundred forty-three pwMS were enrolled between February and May 2019. No patients were excluded due to uphill or downhill route reported on GM®.

Table 1 shows demographic and clinical data of the whole study sample. Education was available for 230/243 (94.6\%) pwMS and the overall mean level of education was $12.4 \pm 3.55$ years. Most (42.4\%) of the patients were treated either with interferon beta-1a (21.0\%) or with dimethyl fumarate $(21.4 \%)$. The clinical phenotype of the 243 pwMS involved in the study was the following: 1 clinically isolated syndrome (0.5\%), 210 relapsing-remitting (RR, 86.5\%), 23 secondary progressive (SP, $9.2 \%$ ), 9 primary progressive (PP, 3.8\%).

\section{Perceived EDSS (pEDSS) and GM ${ }^{\circledast}$ EDSS (gmEDSS)}

Based on the data collected concerning pAS and gmAS, we calculated the pEDSS and the gmEDSS, respectively, as may be seen in Table 1 .

pEDSS and gmEDSS coincided in 169 pwMS and differed in 74/243 pwMS (30.45\%) as shown in Fig. 1.

Due to the weight of other FS on EDSS calculation, only in 7 pwMS, the EDSS calculation was unaffected by the AS results. Comparison between pAS and gmAS was reported in supplementary material.

Based on the EDSS calculated with the pAS (pEDSS) and the EDSS calculated with the gmAS (gmEDSS) (weighted kappa: 0.82; almost perfect agreement), the results can be stratified into three subgroups: (1) pwMS with pEDSS coinciding with gmEDSS; (2) pwMS with pEDSS lower than gmEDSS; (3) pwMS with pEDSS higher than gmEDSS. In each of these subgroups, pwMS with mild and moderate perceived disability were included (Fig. 1).

PwMS with pEDSS that coincided with gmEDSS were significantly younger either than pwMS with pEDSS lower than gmEDSS $(37.5 \pm 12.14$ vs $45.03 \pm 12.16 ; p=0.001)$ or than pwMS with pEDSS higher than gmEDSS $(37.5 \pm 12.14$ vs $45.02 \pm 10.60 ; p=0.001)$. The three groups did not differ as regards education level (Kruskal-Wallis $p=0.607$ ) but they were different for DD (Kruskal-Wallis $p=0.007$ ). In particular, pwMS with pEDSS that coincided with gmEDSS had a significantly shorter DD (median: 7, Q1-Q3: 3-12) than pwMS with pEDSS higher than gmEDSS (median: 11, Q1-Q3: 6.8-18.3; Mann-Whitney Bonferroni adjusted $p=0.003)$.

Moreover, the three groups $(\mathrm{pEDSS}=\mathrm{gmEDSS}$, pEDSS $<$ gmEDSS, and pEDSS $>$ gmEDSS) differed greatly for FSS scores (Kruskal-Wallis $p=0.0001$ ) and for PHQ-9 scores (Kruskal-Wallis $p=0.0001$ ). Post-hoc comparisons (Table 1) showed that pwMS with pEDSS that was in line with gmEDSS had significantly lower FSS scores compared to pwMS with pEDSS higher than gmEDSS (Mann-Whitney Bonferroni adjusted $p<0.001)$ and to pwMS with pEDSS lower than gmEDSS (Mann-Whitney Bonferroni adjusted $p<0.001$ ).

Similarly, pwMS with pEDSS that coincided with gmEDSS had significantly lower PHQ-9 scores than pwMS with pEDSS higher than gmEDSS (median: 11.5, Q1-Q3: 5-16; Mann-Whitney Bonferroni adjusted $p<0.001$ ) and than pwMS with pEDSS lower than gmEDSS (Mann-Whitney Bonferroni adjusted $p<0.001$ ) (Table 1). 
Table 1 Whole population and subgroups stratified based on agreement between pEDSS and gmEDSS; demographic and clinical feature

\begin{tabular}{|c|c|c|c|c|c|c|}
\hline & & Total $(n=243)$ & $\begin{array}{l}\text { pEDSS }=\text { gmEDSS } \\
(n=169)\end{array}$ & $\begin{array}{l}\text { pEDSS }<\text { gmEDSS } \\
(n=32)\end{array}$ & $\begin{array}{l}\text { pEDSS }>\text { gmEDSS } \\
(n=42)\end{array}$ & \\
\hline Variable & & & & & & $P$ value \\
\hline Sex & & & & & & \\
\hline Female (n; \%) & & $173,00(71,19)$ & $118(69,82)$ & $24(75 \%)$ & $31(73.81)$ & 0.771 \\
\hline Male (n; \%) & & $70,00(28,81)$ & $51(30,18)$ & $8(25 \%)$ & $11(26.19)$ & \\
\hline Age & & & & & & \\
\hline Mean, sd & & $39,77(12,35)$ & $37,46(12,14)$ & 45.03 (12.16) & $45.02+10.60$ & $<0.001$ \\
\hline Median (Q1-Q3) & & $39(30-48)$ & $37(27-45)$ & $45,5(37,5-47,5)$ & $44(39-51)$ & \\
\hline Disease type & & & & & & \\
\hline CIS (n; \%) & & $1,00(0,41)$ & $1(0,59)$ & $0(0)$ & $0(0)$ & $<0.001$ \\
\hline $\mathrm{RR}(\mathrm{n} ; \%)$ & & $210,00(86,78)$ & $156(92,31)$ & $20(62,50)$ & $34(82,93)$ & \\
\hline $\mathrm{SP}(\mathrm{n} ; \%)$ & & $23,00(9,09)$ & $10(5,92)$ & $7(21,88)$ & $5(12,20)$ & \\
\hline $\mathrm{PP}(\mathrm{n} ; \%)$ & & $9,00(3,72)$ & $2(1,18)$ & $5(15,62)$ & $2(4,88)$ & \\
\hline Disease duration & & & & & & \\
\hline & mean, sd & $9,60(7,83)$ & $8,68(6,70)$ & $9.78(10.28)$ & $13,16(9,01)$ & 0.007 \\
\hline & median (Q1-Q3) & $7(3-13)$ & $7(3-12)$ & $5,5(3-13)$ & $11(6.8-18.3)$ & \\
\hline Ongoing therapy & & & & & & \\
\hline & $\mathrm{GA}(\mathrm{n} ; \%)$ & $19,00(7,82)$ & $13(7,69)$ & $3(9,38)$ & $3(7.14)$ & \\
\hline & $\operatorname{INF}(\mathrm{n} ; \%)$ & $51,00(20,99)$ & $38(22,49)$ & $7(21,88)$ & $6(14.29)$ & \\
\hline & $\operatorname{DMF}(\mathrm{n} ; \%)$ & $52,00(21,40)$ & $39(23,08)$ & $5(15,62)$ & $8(19.05)$ & \\
\hline & TRF (n; \%) & $16,00(6,58)$ & $6(3,55)$ & $6(18,75)$ & $4(9.52)$ & \\
\hline & FTY (n; \%) & $46,00(18,93)$ & $37(21,89)$ & $0(0)$ & $9(21.43)$ & \\
\hline & NTZ (n; \%) & $36,00(14,81)$ & $25(14,79)$ & $4(12,50)$ & $7(16.67)$ & \\
\hline & $\operatorname{ALZ}(\mathrm{n} ; \%)$ & $10,00(4,12)$ & $5(2,96)$ & $4(12,50)$ & $1(2.38)$ & \\
\hline & None $(\mathrm{n} ; \%)$ & $13,00(5,35)$ & $6(3,55)$ & $3(9,38)$ & $4(9.52)$ & \\
\hline FSS & & & & & & \\
\hline & mean, sd & $30,63(16,09)$ & $26.68(14.27)$ & $39.65625(17.80)$ & $39,61(15,60)$ & $<0.001$ \\
\hline & median (Q1-Q3) & $3016-43$ & $24(14-37)$ & $44,5(21-55,5)$ & $38(30-53)$ & \\
\hline PHQ9 & & & & & & \\
\hline & mean, sd & $7,52(6,07)$ & $5.86(4.92)$ & 11.1875 (7.168198) & $11.35714(6.49135)$ & $<0.001$ \\
\hline & median (Q1-Q3) & $6(3-11)$ & $5(2-8)$ & $12(5-15,5)$ & $11.5(5-16)$ & \\
\hline pEDSS & & & & & & \\
\hline & $<=3,5(\mathrm{n} ; \%)$ & $142(58.68)$ & $130(76,92)$ & $11(34,38)$ & $1(2,44)$ & $<0.001$ \\
\hline & $>=4(\mathrm{n} ; \%)$ & $101(41.32)$ & $39(23,08)$ & $21(65.62)$ & $41(97,56)$ & \\
\hline
\end{tabular}

GA glatiramer acetate, INF interferon, $D M F$ dimethyl fumarate, TRF teriflunomide, $F T Y$ fingolimod, NTZ natalizumab, ALZ alemtuzumab, EDSS Expanded Disability Status Scale, pEDSS perceived EDSS, gmEDSS Google EDSS, FSS Fatigue Severity Scale, PHQ-9 Patient Health Questionnaire-9

Finally, pwMS with pEDSS that coincided with gmEDSS were more likely to have a pEDSS score $<=3.5(130$; $76.92 \%$ ) compared to both pwMS with pEDSS lower than gmEDSS $(11 ; 34.38 \%)$ and pwMS with pEDSS higher than gmEDSS $(1 ; 2.44 \%)($ Chi-square $p<0.001)$ (Table 1$)$.

\section{ObjectiveAS (obAS)}

Demographic and clinical data from the subgroup of pwMS with obAS calculation are shown in Table 2.

This group consisted of 75 pwMS (58 F [77.3\%]; $17 \mathrm{M}$ [22.7\%]). The mean level of education was $11.1 \pm 2.69$ years (median: 13; Q1-Q3: 8-13 years). The mean age was $41.3+11.71$ years (median: $41 ; \mathrm{Q} 1-\mathrm{Q} 3=32-47$ ). The mean DD was $10.7 \pm 8.40$ years (median: 9; Q1-Q3: 4-14). In this subgroup, the obAS and gmAS are more frequently coincident (45 patients; 60\%) either than obAS and pAS (34 patients; $45.3 \%$ ) or than pAS and gmAS (39 patients; 52\%).

\section{Factors influencing EDSS variation (pEDSS different from gmEDSS)}

Logistic regression (Table 3) models showed that the progressive phenotypes $(p=0.03)$ as well as higher FSS 


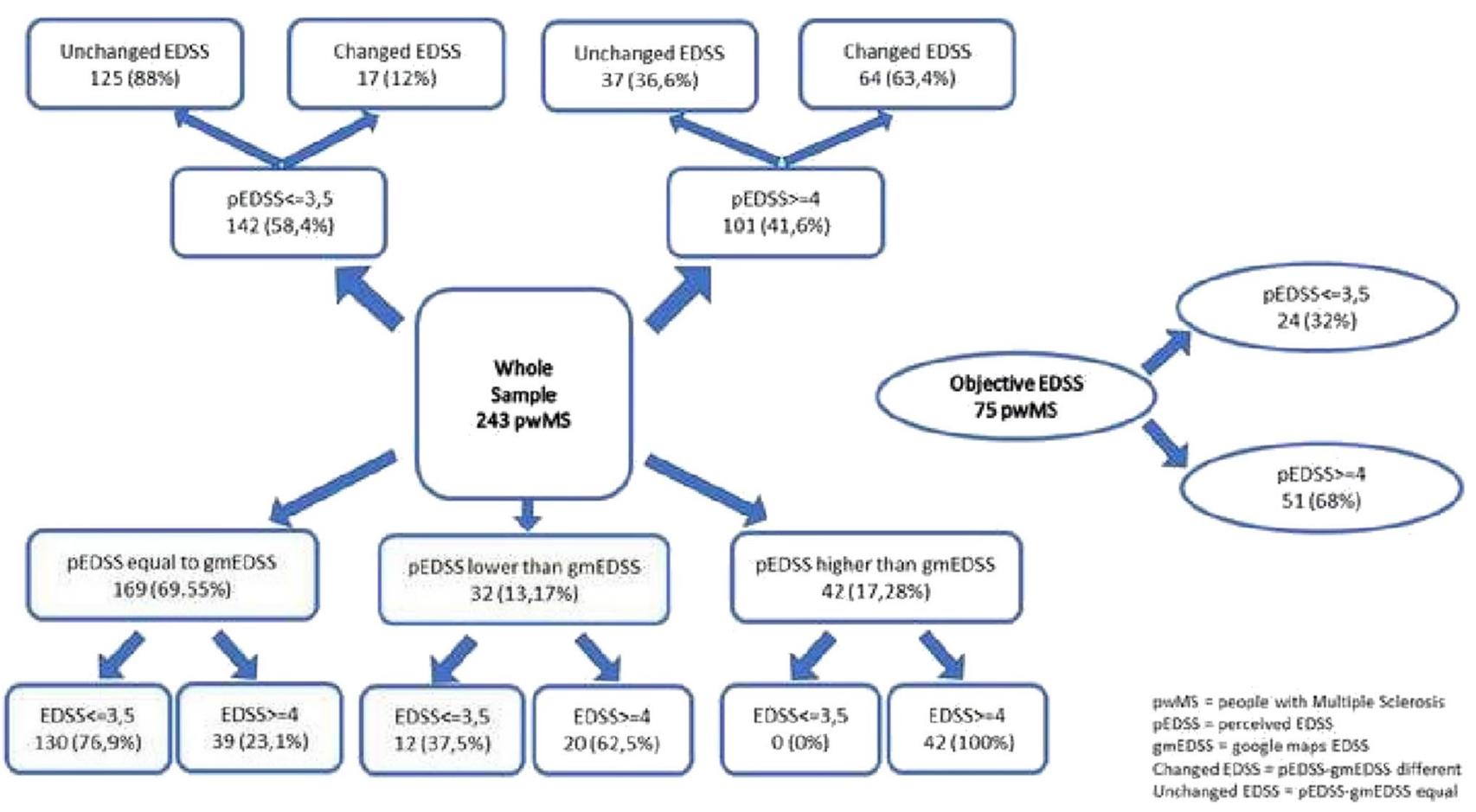

Fig. 1 General flowchart on Expanded Disability Status Scale (EDSS) results

( $p=0.01)$ and PHQ-9 $(p=0.002)$ scores were associated with the discrepancy between pEDSS and gmEDSS.

\section{Discussion}

In neurology clinical practice, the evaluation of walking ability is frequently based on the question "how long can you walk without rest?" In the MS field, the EDSS requires patients to walk at least $500 \mathrm{~m}$ to obtain the AS. However, there are a number of limitations coming from both approaches. There are many variables that may influence the actual measurement of walking distance: (1) time and space constraints limiting an optimal examination; (2) daily variations in fatigue and overall functioning; (3) concomitant diseases and treatments. The same applies to self-reported measurements: (1) inaccuracy in providing an exact estimate of distances [23]; (2) depressed mood [24]; (3) being fatigued at question time [25]; (4) level of education [26]; and (5) impairment of mental functions [27].

We used the GM® application to evaluate the MWD in pwMS and therefore investigated whether GM® might be used as an objective tool to evaluate the AS in pwMS and the potential implications on EDSS scoring accuracy.

Our results showed that one-third of our patients indicated a pAS different from the gmAS. These data are consistent with a previous study showing that pwMS are inaccurate at estimating distances [9], suggesting that the estimates of walking distances covered are not reliable indicators for disability assessment. We found that the discrepancies between pAS and gmAS are more frequent in the subgroup of pwMS with moderate disability (63\%), indicating a less reliable estimate of MWD in more disabled patients and consequently prompting clinicians to improve disability assessment to monitor disease course in clinical practice. In particular, once the EDSS milestone of 4.0 is reached, further progression in the EDSS score can be independent of decline or improvement in the other functional systems and determined solely by the MWD a MS patient can usually walk. Therefore, differences in MWD are very important in pwMS with moderate disability and should be monitored closely and carefully to improve EDSS assessment. Unfortunately, routine clinical examination for pwMS does not include an objective evaluation of the AS and the EDSS score is calculated based on what patients report to their neurologists.

To partially overcome the risk of inaccurate estimates of the MWD, we used GM® application to calculate the gmAS and the gmEDSS. Those pwMS with pEDSS corresponding to the gmEDSS were found to be younger and with a shorter disease duration (DD) but, above all, these patients had significantly lower FSS and PHQ-9 scores. A previous study reported that age does not influence the patient estimation of MWD [28]. Our data 
Table 2 obAS population; demographic and clinical features

Variable

Age

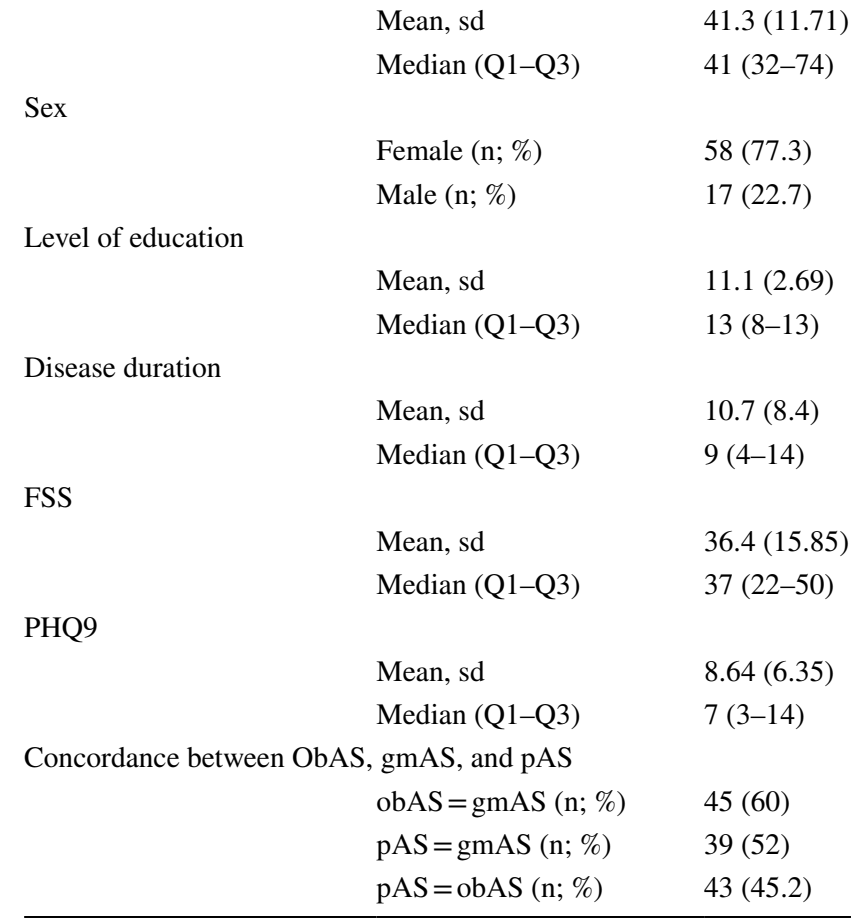

Abbreviations: $p A S$ perceived Ambulation Score; $p E D S S$ perceived Expanded Disability Status Scale; gmAS Google Maps Ambulation Score; gmEDSS Google Maps Expanded Disability Status Scale; $o b A S$ actual (objective) Ambulation Score; obEDSS actual (objective) Expanded Disability Status Scale; FSS Fatigue Severity Scale; $P H Q$ 9 Patient Health Questionnaire-9

confirmed this result but showed a better correspondence between pEDSS and gmEDSS in younger pwMS with significantly lower DD, probably reflecting a milder disease [29]. In pwMS where pAS and gmAS were not concordant, we found that $42 \%$ had a pAS lower than the gmAS and $58 \%$ had a pAS higher than the gmAS.
Distinguishing these subgroups of pwMS with mild and moderate disability, we found that pwMS with a moderate disability tend to overestimate MWD (with respect to GM® output). In these groups, pwMS with unchanged EDSS (pEDSS $=$ gmEDSS) and those with discrepant EDSS (pEDSS $<$ or $>$ gmEDSS), we found that progressive phenotype and higher scores in FSS and PHQ-9 increased the probability of inaccurate estimates of MWD and therefore belonged to the discrepant group. These data are consistent with the findings of Skjearbak et al. [30], showing that the extent of misclassification of MWD increases in pwMS with fatigability and progressive phenotype and that depressive symptoms are related to self-perception of MWD; these findings emphasize the need to improve disability assessment especially in pwMS with moderate disability. Furthermore, a subgroup analysis conducted on the 75 patients in whom the obAS was calculated showed that GM® seems to effectively improve the accuracy of the MWD. According to our results, $\mathrm{GM}{ }^{\circledR}$ technology may represent a feasible tool to measure MWD increasing the agreement with objective measures. Ideally, a large-scale clinical trial should be focused on pwMS who are at higher risk of making false estimates of MWD (i.e., fatigued and depressed) or in those pwMS with a moderate disability or transitional forms of the disease where the prompt close monitoring of disability progression is necessary for therapy adjustment and ultimately to improve pwMS management. The results of the subgroup obAS/obEDSS strengthen the hypothesis that GM $®$ may represent an objective tool to evaluate the AS in pwMS, and could result in greater accuracy of EDSS scoring.

In conclusion, our results suggest that GM® application may be easily and accurately used in a real-life clinical setting to improve the evaluation of the AS and the related EDSS, thus making it also suitable to overcome the difficulties imposed by the ongoing health-care restrictions due to the pandemic.

Table 3 Univariate and multivariate logistic models: factors influencing the discrepancy between pEDSS and gmEDSS

\begin{tabular}{|c|c|c|c|c|c|c|c|c|c|c|c|c|c|c|}
\hline \multirow[t]{2}{*}{ Variables } & \multicolumn{2}{|c|}{ Model I } & \multicolumn{2}{|c|}{ Model II } & \multicolumn{2}{|c|}{ Model III } & \multicolumn{2}{|c|}{ Model IV } & \multicolumn{2}{|c|}{ Model V } & \multicolumn{2}{|c|}{ Model VI } & \multicolumn{2}{|c|}{ Model VII } \\
\hline & OR & $P$ value & OR & $P$ value I & OR & $P$ value & OR & $P$ value & OR & $P$ value & OR & $P$ value & OR & $P$ value \\
\hline Disease type & 3.34 & $<0.001$ & & & & & & & & & & & 2.8 & 0.03 \\
\hline Age, y & & & 1.05 & $<0.001$ & & & & & & & & & 1.02 & 0.164 \\
\hline Education, years & & & & & 0.9 & 0.381 & & & & & & & 0.95 & 0.321 \\
\hline DD & & & & & & & 1.04 & 0.007 & & & & & 1.07 & 0.208 \\
\hline FSS & & & & & & & & & 1.05 & $<0.001$ & & & 1.06 & 0.01 \\
\hline PHQ-9 & & & & & & & & & & & 1.16 & $<0.001$ & 1.17 & 0.002 \\
\hline
\end{tabular}

Abbreviations: $P P$ primary progressive multiple sclerosis; $S P$ secondary progressive multiple sclerosis; $R R$ relapsing-remitting multiple sclerosis; FSS Fatigue Severity Scale; $P H Q-9$ Patient Health Questionnaire-9 
Supplementary Information The online version contains supplementary material available at https://doi.org/10.1007/s10072-021-05389-7.

\section{Declarations}

Ethical approval The Ethical Commitee of University of Campania Luigi Vanvitelli (Coordinator Center) approved the project and the protocol numner is 0014460/i.

Competing interest The authors declare no competing interests.

\section{References}

1. Chatterji P, Li Y (2021) Effects of the COVID-19 pandemic on outpatient providers in the United States. Med Care 59(1):58-61

2. Moccia M, Lanzillo R, Brescia Morra V et al (2020) Assessing disability and relapses in multiple sclerosis on tele-neurology. Neurol Sci 41:1369-1371

3. Bombaci A, Abbadessa G, Trojsi F et al (2021) Telemedicine for management of patients with amyotrophic lateral sclerosis through COVID-19 tail. Neurol Sci 42(1):9-13

4. Bove R, Bevan C, Crabtree E, Zhao C, Gomez R, Garcha P, Morrissey J, Dierkhising J, Green AJ, Hauser SL, Cree BA, Wallin MT, Gelfand JM (2019) Toward a low-cost, in-home, telemedicine-enabled assessment of disability in multiple sclerosis. Mult Scler 25(11):1526-1534

5. Abbadessa G, Lavorgna L, Miele G et al (2021) Assessment of multiple sclerosis disability progression using a wearable biosensor: a pilot study. J Clin Med 10(6):1160

6. Kurtzke JF (1983) Rating neurologic impairment in multiple sclerosis: an expanded disability status scale (EDSS). Neurology 33(11):1444-1452

7. Kruidenier LM, Nicolai SPA, Willidgendael EM et al (2009) Functional claudication distance: a reliable and valid measurement to assess functional limitation in patients intermittent claudication. BMC Cardiovasc Disord 2(9):9

8. Sharrack B, Hughes RCA (1997) Reliability of distance estimation by doctors and patients: cross sectional study. BMJ 315:1652-1654

9. Hohart J, Lamping D, Fitzpatrick R et al (2001) The multiple sclerosis impact scale: a new patients based outcome measures. Brain 124:962-973

10 PiriÇinar B, GüvenYorgun K (2018) What we learned from the history of multiple sclerosis measurement: expanded disability status scale. Arch Neuropsychiatry 55(Supplement 1):69-75

11 Sparaco M, Lavorgna L, Conforti R et al (2018) The role of wearable devices in multiple sclerosis. Mult Scler Int 2018:7627643

12 Krupp LB, LaRocca NG, Muir-Nash J et al (1989) The fatigue severity scale. Application to patients with multiple sclerosis and systemic lupus erythematosus. Arch Neurol 46:1121-1123

13. Hind D, Kaklamanou D, Beever D et al (2016) The assessment of depression in people with multiple sclerosis: a systematic review of psychometric validation studies. BMC Psychiatry 16:278

14. Goldman MD, Motl RW, Rudick RA (2010) Possible clinical outcome measures for clinical trials in patients with multiple sclerosis. Ther Adv Neurol Disord 3:229-239
15. Confavreux C, Vukusic S, Adeline P (2003) Early clinical predictors and progression of irreversible disability in multiple sclerosis: an amnesic process. Brain 126:770-782

16. Confavreux C, Vukusic S, Moreau T, Adeleine P (2000) Relapses and progression of disability in multiple sclerosis. N Eng J Med 343:1430-1438

17 Kimmelman J, Mogil JS, Dirnagl U (2014) Distinguishing between exploratory and confirmatory preclinical research will improve translation. Plos Biol 12(5):e1001863

18. Arain M, Campbell MJ, Cooper CL, Lancaster GA (2010) What is a pilot or feasibility study? A review of current practice and editorial policy. BMC Med Res Methodol 10:67

19. Koo TK, Li MY (2016) A guideline of selecting and reporting intraclass correlation coefficients for reliability research. J Chiropr Med 15(2):155-163

20. Mukaka MM (2012) A guide to appropriate use of Correlation coefficient in medical research MM Mukaka. Malawi Med J 24(3):69-71

21. Mandrekar JN (2011) Measures of interrater agreement. J Thorac Oncol 6(1):6-7

22. Dong Y, Peng CY (2013) Principled missing data methods for researchers. Springerplus 2(1):222. Published 2013 May 14. https://doi.org/10.1186/2193-1801-2-222.

23. Herbolsheimer F, Riepe M, Peter R (2018) Cognitive function and the agreement between self-reported and accelerometer-accessed physical activity. BMC Geriatr 18:56

24. Kalron A, Aloni R (2018) Contrasting relationship between depression, quantitative gait characteristics and self-report walking difficulties in people with multiple sclerosis. Mult Scler Relat Disord 19:1-5

25. Dalgas U, Langeskov-Christensen M, Skjerbæk AG (2018) Is the impact of fatigue related to walking capacity and perceived ability in persons with multiple sclerosis? A multicenter study. J Neurol Sci 387:179-186

26. Berger W, Payne MWC, Morrow SA (2017) Self reported maximum walking distance in persons with MS may affect the EDSS. Neurol Sci 379:77-80

27. Neil BA, Kenneth EG, Darryl GT et al (2000) Self-Reported walking ability predicts functional mobility performance in frail older adults. JAGS 48:1408-1413

28. Giantomaso T, Makowsky L, Ashworth N et al (2003) The validity of patient and physician estimate of walking distance. Clin Rehabil 17:394-401

29 Minden SL, Frankel D, Hadden L et al (2004) Disability in elderly people with multiple sclerosis: an analysis of baseline data from the Sonya Slifka longitudinal multiple sclerosis study. Neurorehabilitation 19(1):55-67

30. Skjerbæk AG, Boesen F, Petersen T, Rasmussen PV, Stenager E, Nørgaard M, Feys P, Kjeldgaard-Jørgensen ML, Hvid LG, Dalgas U. (2019) Can we trust self-reported walking distance when determining EDSS scores in patients with multiple sclerosis? The Danish MS hospitals rehabilitation study. Mult Scler. 25(12):16531660. https://doi.org/10.1177/1352458518795416

Publisher's note Springer Nature remains neutral with regard to jurisdictional claims in published maps and institutional affiliations. 


\section{Authors and Affiliations}

\begin{tabular}{|c|c|c|}
\hline \multicolumn{3}{|c|}{$\begin{array}{l}\text { Luigi Lavorgna }^{1} \text { (D) Pietro laffaldano }{ }^{2} \cdot \text { Gianmarco Abbadessa }^{3} \cdot \text { Roberta Lanzillo }^{4} \cdot \text { Sabrina Esposito }^{5,6} \text {. } \\
\text { Domenico Ippolito }^{7} \cdot \text { Maddalena Sparaco }^{8} \cdot \text { Simone Cepparulo }^{8} \cdot \text { Giacomo Lus }^{3} \cdot \text { Rosa Viterbo }^{2} \cdot \text { Marinella } \text { Clerico }^{9} \\
\text { Francesca Trojsi }^{10} \cdot \text { Paolo Ragonese }^{11} \cdot \text { Giovanna Borriello }^{12} \cdot \text { Elisabetta Signoriello }^{3} \cdot \text { Raffaele Palladino }^{13} \cdot \\
\text { Marcello Moccia }^{4} \cdot \text { Francesco Brigo }^{14} \cdot \text { Maria Troiano }^{2} \cdot \text { Gioacchino Tedeschi }^{15} \cdot \text { Simona Bonavita }^{8}\end{array}$} \\
\hline $\begin{array}{l}\text { Pietro Iaffaldano } \\
\text { pietro.iaffaldano@uniba.it }\end{array}$ & \multirow[t]{2}{*}{1} & \\
\hline Gianmarco Abbadessa & & $\begin{array}{l}\text { and Surgical Sciences, AOU-University of Campania "Luigi } \\
\text { Vanvitelli", Piazza Miraglia 2, } 80138 \text { Naples, Italy }\end{array}$ \\
\hline $\begin{array}{l}\text { Roberta Lanzillo } \\
\text { roberta.lanzillo@unina.it }\end{array}$ & 2 & $\begin{array}{l}\text { Department of Basic Medical Sciences, Neurosciences } \\
\text { and Sense Organs, University of Bari “Aldo Moro”, Piazza } \\
\text { G. Cesare 11, 70124 Bari, Italy }\end{array}$ \\
\hline $\begin{array}{l}\text { Sabrina Esposito } \\
\text { sabrina.esposito1@ unicampania.it }\end{array}$ & \multirow[t]{2}{*}{3} & $\begin{array}{l}\text { Second Division of Neurology, Department of Advanced } \\
\text { Medical and Surgical Sciences, University of Campania }\end{array}$ \\
\hline \multirow{2}{*}{$\begin{array}{l}\text { Domenico Ippolito } \\
\text { mimmoip88@gmail.com }\end{array}$} & & "Luigi Vanvitelli", Via Sergio Pansini 5, 80131 Naples, Italy \\
\hline & \multirow[t]{2}{*}{4} & Multiple Sclerosis Clinical Care and Research Centre, \\
\hline $\begin{array}{l}\text { Maddalena Sparaco } \\
\text { ms.86@ hotmail.it }\end{array}$ & & $\begin{array}{l}\text { Department of Neuroscience, Reproductive Science } \\
\text { and Odontostomatology, Federico II University, Naples, Italy }\end{array}$ \\
\hline $\begin{array}{l}\text { Simone Cepparulo } \\
\text { cepparulo.s@gmail.com }\end{array}$ & \multirow[t]{2}{*}{5} & $\begin{array}{l}\text { Department of Advanced Medical and Surgical Sciences, } \\
\text { University of Campania "Luigi Vanvitelli", Piazza Miraglia }\end{array}$ \\
\hline \multirow{2}{*}{$\begin{array}{l}\text { Giacomo Lus } \\
\text { giacomo.lus@unina2.it }\end{array}$} & & 2, 80138 Naples, Italy \\
\hline & 6 & Department of Neuroscience, Psychology, Drug Research \\
\hline Rosa Viterbo & \multirow[t]{2}{*}{7} & First Division of Neurology, Department of Advanced \\
\hline $\begin{array}{l}\text { Marinella Clerico } \\
\text { marinella.clerico@unito.it }\end{array}$ & & $\begin{array}{l}\text { Medical and Surgical Sciences, AOU-University } \\
\text { of Campania "Luigi Vanvitelli", P.zza Miraglia 2, Naples, } \\
\text { Italy }\end{array}$ \\
\hline $\begin{array}{l}\text { Francesca Trojsi } \\
\text { francesca.trojsi@unicampania.it }\end{array}$ & \multirow[t]{2}{*}{8} & $\begin{array}{l}\text { Second Division of Neurology, Department of Advanced } \\
\text { Medical and Surgical Sciences, MRI Research Center }\end{array}$ \\
\hline $\begin{array}{l}\text { Paolo Ragonese } \\
\text { paolo.ragonese@unipa.it }\end{array}$ & & $\begin{array}{l}\text { SUN-FISM, AOU-University of Campania "Luigi } \\
\text { Vanvitelli", via Sergio Pansini 5, } 80131 \text { Naples, Italy }\end{array}$ \\
\hline $\begin{array}{l}\text { Giovanna Borriello } \\
\text { giovanna.borriello@gmail.com }\end{array}$ & 9 & $\begin{array}{l}\text { Clinical and Biological Sciences Department, University } \\
\text { of Torino, Turin, Italy }\end{array}$ \\
\hline $\begin{array}{l}\text { Elisabetta Signoriello } \\
\text { elisabetta.signoriello@gmail.com }\end{array}$ & \multirow[t]{2}{*}{10} & $\begin{array}{l}\text { First Division of Neurology, Department of Advanced } \\
\text { Medical and Surgical Sciences, AOU-University }\end{array}$ \\
\hline $\begin{array}{l}\text { Raffaele Palladino } \\
\text { raffaele.palladino@unina.it }\end{array}$ & & $\begin{array}{l}\text { of Campania “Luigi Vanvitelli”, P.zza Miraglia 2, } \\
80138 \text { Naples, Italy }\end{array}$ \\
\hline $\begin{array}{l}\text { Marcello Moccia } \\
\text { moccia.marcello@gmail.com }\end{array}$ & 11 & $\begin{array}{l}\text { Department of Experimental, Biomedicine and Clinical } \\
\text { Neurosciences, University of Palermo, } 90129 \text { Palermo, Italy }\end{array}$ \\
\hline Francesco Brigo & 12 & S. Andrea Hospital, Sapienza Rome University, Rome, Italy \\
\hline dr.francescobrigo@gmail.com & 13 & Federico II University, Naples, Italy \\
\hline $\begin{array}{l}\text { Maria Troiano } \\
\text { maria.trojano@uniba.it }\end{array}$ & 14 & $\begin{array}{l}\text { UOC di Neurologia, Ospedale Di Merano (SABES-ASDAA), } \\
\text { Via Rossini, 5, } 39012 \text { Merano-Meran, BZ, Italy }\end{array}$ \\
\hline $\begin{array}{l}\text { Gioacchino Tedeschi } \\
\text { gioacchino.tedeschi@unicampania.it }\end{array}$ & \multirow[t]{2}{*}{15} & $\begin{array}{l}\text { First Division of Neurology, Department of Advanced } \\
\text { Medical and Surgical Sciences, MRI Research Center }\end{array}$ \\
\hline $\begin{array}{l}\text { Simona Bonavita } \\
\text { simona.bonavita@unicampania.it }\end{array}$ & & $\begin{array}{l}\text { SUN-FISM, AOU, University of Campania "Luigi } \\
\text { Vanvitelli”, Piazza Miraglia 2, } 80138 \text { Naples, Italy }\end{array}$ \\
\hline
\end{tabular}

